\title{
Caspase-dependent mitochondrial apoptotic pathway is involved in astilbin-mediated cytotoxicity in breast carcinoma cells
}

\author{
XIAOQI SUN ${ }^{1}$, HONG ZHANG $^{1}$, YINGYU ZHANG ${ }^{1}$, QI YANG $^{2}$ and Shujie Zhao ${ }^{3}$ \\ ${ }^{1}$ Faculty of Clinical Medicine, Changchun Medical College, Changchun, Jilin 130031; ${ }^{2}$ Department of Imaging, \\ The First Hospital of Jilin University, Jilin University, Changchun, Jilin 130021; ${ }^{3}$ Department of Endocrinology, \\ The Second Hospital of Jilin University, Jilin University, Changchun, Jilin 13000, P.R. China
}

Received March 14, 2018; Accepted July 23, 2018

DOI: $10.3892 /$ or.2018.6602

\begin{abstract}
Astilbin exhibits several pharmacological activities, including hypoglycemic, anti-oxidant and anti-inflammatory properties. The aim of the present study was to investigate the pro-apoptotic activities of astilbin on breast cancer in cells and mice. It was demonstrated that astilbin significantly reduced the cell viability, increased the cell apoptosis rate, suppressed the migration ability, caused the dissipation of the mitochondrial membrane potential and induced the overaccumulation of intracellular reactive oxygen species in MCF-7 and MDA-MB231 cells after 12 or $24 \mathrm{~h}$ of exposure. Data obtained from western blotting suggested that astilbin suppressed the expression levels of B-cell lymphoma 2 (Bcl-2), while it increased the expression levels of cleaved caspase- $3,-8$ and -9 , and Bcl-2associated $\mathrm{X}$ protein in breast carcinoma cells. Furthermore, astilbin inhibited the growth of MCF-7-xenografted tumors in nude mice without influencing their bodyweights or organ (liver, spleen and kidney) functions. Additionally, astilbin enhanced the expression of pro-apoptotic proteins and suppressed the expression of anti-apoptotic proteins in tumor tissues. All these results revealed that astilbin exhibits pro-apoptotic properties in breast carcinoma cells via modulation of the caspase-dependent pathway, which highlights the feasibility of astilbin as a candidate agent for breast cancer treatment.
\end{abstract}

\section{Introduction}

Breast cancer is the most common cancer among women (1), representing $25 \%$ of all cancer diagnoses (2). This disease and its associated social impacts have become a major health problem worldwide. Standard chemotherapy and radiotherapy

Correspondence to: Professor Shujie Zhao, Department of Endocrinology, The Second Hospital of Jilin University, Jilin University, 218 Ziqiang Street, Changchun, Jilin 13000, P.R. China E-mail: zsjdr@sina.com

Key words: astilbin, breast carcinoma, apoptosis, mitochondria, caspase protocols for breast cancer have been developed over the years; however, several subtypes of breast cancer have been identified that exhibit different responses to these therapeutic regimens, which further limits the agent and treatment options. In this scenario, the identification of more effective alternative therapies or novel drugs, targeting one or more tumor-specific biomarkers that define the more aggressive breast cancer subtypes, is urgently required.

Apoptosis, an energy-dependent process of programmed cell death undertaken by living cells, is associated with internucleosomal DNA fragmentation, chromatin condensation and cell shrinkage $(3,4)$. Cell apoptosis is initiated by extracellular and intracellular signals via two main pathways, including the death receptor-mediated pathways and the mitochondrial apoptosis $(5,6)$. During mitochondrial apoptosis, the activation of the caspase family, including casapase- $3,-8$ and -9 , serves a central role (7). For instance, the overaccumulation of intracellular reactive oxygen species (ROS) enhances the dissipation of the mitochondrial membrane potential (MMP; also known as $\Delta \psi \mathrm{m}$ ), and the resulting increased permeability of the mitochondria further enhances the excessive release of ROS into the cytoplasm, as well as of cytochrome $c$, which triggers the activation of caspase-9 $(8,9)$. In addition, the auto-catalytic activation of procasapase- 8 can cleave Bid into truncated Bid, which initiates the mitochondrial apoptotic pathway $(10,11)$. The activation of caspase- 8 or -9 is followed by that of caspase- 3 , which initiates the cell death process and can serve as an index of cell apoptosis (12).

Recently, various natural compounds have been proven to be valuable sources of alternative antitumor agents, due to their greater efficacy and fewer adverse effects (13). One such compound is $18-\beta-$ glycyrrhetinic acid, which is mainly obtained from Glycyrrhiza plants and displays pro-apoptotic properties in pituitary adenoma cells via the regulation of ROS and mitogen-activated protein kinases (MAPKs) (14). It has also been reported that carnosic acid induces the apoptosis of hepatocellular carcinoma cells via an ROS-mediated mitochondrial apoptosis pathway (15). Astilbin is a flavonoid that is commonly found in various herbal medicines and foods, such as Smilax glabra Roxb., Sarcandra glabra (Thunb.) Nakai and grapes (16), and its structure is displayed in Fig. 1. According to previous studies, astilbin exhibits anti-arthritic (17), antiinflammatory (18) and anti-oxidative (19) effects. Although 
the antitumor properties of astilbin have been described (20), its pro-apoptotic effect on breast cancer has not yet been reported.

In the present study, the pro-apoptotic effects of astilbin on breast cancer were investigated in MCF-7 and MDA-MB-231 cells, and in nude mice bearing MCF-7-xenografted tumors. Astilbin exhibited cytotoxicity, mainly through the modulation of the caspase-dependent mitochondrial apoptosis pathway.

\section{Materials and methods}

Cell culture. The breast cancer cell lines MDA-MB-231 (ATCC no. HTB-26) and MCF-7 (ATCC no. HTB-22) were obtained from the American Type Culture Collection (ATCC; Manassas, VA, USA). Cells were cultured in Dulbecco's modified Eagle's medium (Invitrogen; Thermo Fisher Scientific, Inc., Waltham, MA, USA) containing $10 \%$ fetal bovine serum (Invitrogen; Thermo Fisher Scientific, Inc.) and supplemented with $100 \mathrm{U} / \mathrm{ml}$ penicillin and $100 \mu \mathrm{g} / \mathrm{ml}$ streptomycin (Invitrogen; Thermo Fisher Scientific, Inc.) under a humidified atmosphere containing $5 \% \mathrm{CO}_{2}$ and $95 \%$ air at $37^{\circ} \mathrm{C}$. The culture medium was changed every other day.

Cell viability assay. 3-(4,5-Dimethylthiazol-2-yl)-2,5diphenyltetrazolium bromide (MTT; Sigma-Aldrich; Merck KGaA, Darmstadt, Germany) assay was applied to detect the cell viability (21). Briefly, samples of $100 \mu \mathrm{l}$ breast carcinoma cells at a density of 50,000 cells $/ \mathrm{ml}$ were seeded into 96 -well plates. After 24- and 48-h treatment with astilbin (CAS no. 29838-67-3; obtained from Shanghai Yuanye Biotechnology Co., Ltd., Shanghai, China) at doses of 0-300 $\mu \mathrm{M}$, MTT was added to each well (final concentration, $0.5 \mathrm{mg} / \mathrm{ml}$ ). The cells were incubated for a further $4 \mathrm{~h}$ at $37^{\circ} \mathrm{C}$ in the dark, and then $100 \mu 1$ dimethyl sulfoxide was added to solubilize the purple formazan crystals. A microplate reader (Bio-Rad Laboratories, Inc., Hercules, CA, USA) was used to detect the absorbance at a wavelength of $490 \mathrm{~nm}$. The half maximal inhibitory concentration $\left(\mathrm{IC}_{50}\right)$ values were calculated by the SPSS version 16.0 software (SPSS, Inc., Chicago, IL, USA).

Cell apoptosis and migration ability assays. In order to examine the cell apoptosis, samples of $2 \mathrm{ml}$ breast carcinoma cells at a density of $2 \times 10^{5}$ cells/ml were seeded into 6 -well plates. After 24-h exposure to astilbin at the doses of 50 and $200 \mu \mathrm{M}$, the cells were collected and stained with propidium iodide and Annexin V (Dead Cell Kit; EMD Millipore, Billerica, MA, USA) for $15 \mathrm{~min}$ at $25^{\circ} \mathrm{C}$ in the dark. A Muse ${ }^{\mathrm{TM}}$ Cell Analyzer from EMD Millipore was applied to analyze the fluorescence intensity.

For determination of the cell migration ability, a wound healing assay was performed. Briefly, the seeded cells were scraped with a 200 pipette tip, and then exposed to astilbin at the doses of 50 and $200 \mu \mathrm{M}$ for $24 \mathrm{~h}$. The distances traveled by the migrating cells were quantified using the Image J $1.48 \mathrm{v}$ software (National Institutes of Health, Bethesda, MD, USA; rsb.info.nih.gov/ij/download.html) to evaluate the cell migratory ability.

Assessment of intracellular ROS levels and MMP. For intracellular ROS level determination, samples of $2 \mathrm{ml}$ breast

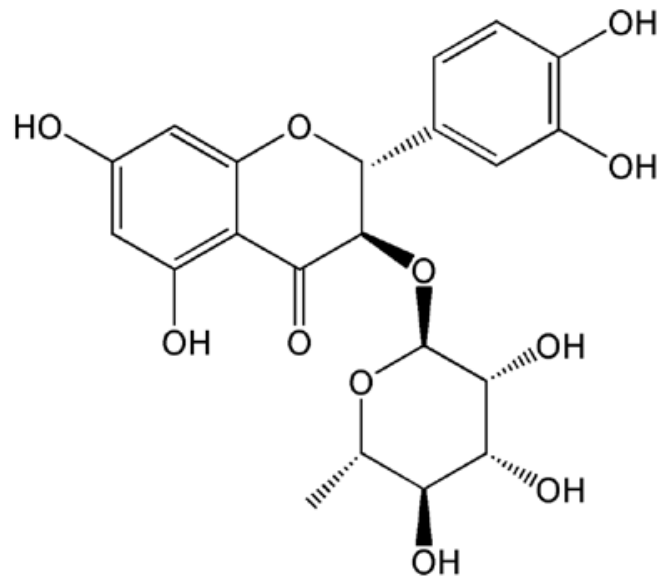

Figure 1. Structural formula of astilbin.

carcinoma cells at a density of $2 \times 10^{5}$ cells $/ \mathrm{ml}$ were seeded into 6 -well plates, and exposed to astilbin at the doses of 50 and $200 \mu \mathrm{M}$ for $12 \mathrm{~h}$. Subsequently, cells were stained for $20 \mathrm{~min}$ with 2,7-dichlorofluorescein diacetate (Sigma-Aldrich; Merck $\mathrm{KGaA})$ at $37^{\circ} \mathrm{C}$ in the dark. The changes in intracellular ROS levels were observed using fluorescent microscopy (magnification, x20; CCD camera, Axio Observer Z1; Carl Zeiss AG, Oberkochen, Germany). The quantitative data were analyzed with the ImageJ software, and are expressed as the green fluorescence intensity.

For MMP determination, the treated cells were stained with $2 \mu \mathrm{M} 5,5$ ',6,6'-tetrachloro-1,1',3,3'-tetraethylbenzimidazolylcarbocyanine iodide (JC-1; Sigma-Aldrich; Merck KGaA) for $15 \mathrm{~min}$ at $37^{\circ} \mathrm{C}$ in the dark. The changes in fluorescence from red to green were detected using fluorescent microscopy (magnification, x20; CCD camera, Axio Observer Z1; Carl Zeiss AG). The quantitative data were analyzed with the ImageJ software, and are expressed as the ratio of red to green fluorescence intensity.

MCF-7 xenograft tumor model. The experimental animal study was approved by the Ethics Committee of Changchun Medical College (approval no. CCMC2016-1201; Changchun, China). In total, 10 male BALB/c athymic nude mice (5-week-old; SCXK 2012-0001) were obtained from the Model Animal Research Center of Nanjing University (Nanjing, China). The mice were maintained on a 12 -h light/dark cycle at $23 \pm 1^{\circ} \mathrm{C}$ with water and food available ad libitum. A sample of $0.15 \mathrm{ml}$ MCF-7 cell suspension at a density of $2 \times 10^{8}$ cells $/ \mathrm{ml}$ was subcutaneously injected into the right-side waist of each nude mouse. After 4 days, the mice were randomly divided into two groups ( $\mathrm{n}=5$ each), and intraperitoneally injected with astilbin (20 mg/kg; $0.3 \mathrm{ml} / \mathrm{mouse})$ or normal saline $(0.3 \mathrm{ml} / \mathrm{mouse})$, respectively, every other day for 14 days. The tumor dimensions were monitored throughout the experiment. The tumor volume $\left(\mathrm{mm}^{3}\right)$ was estimated using the following equation: Volume $=$ length $\times(\text { width })^{2} \times 0.5$. Finally, the mice were sacrificed by administration of $200 \mathrm{mg} / \mathrm{kg}$ pentobarbital. Liver, spleen, kidney and tumor tissues were carefully dissected from each mouse for western blot analysis. The protocol of the animal experiments was followed as described in previous studies (22-24). 
A
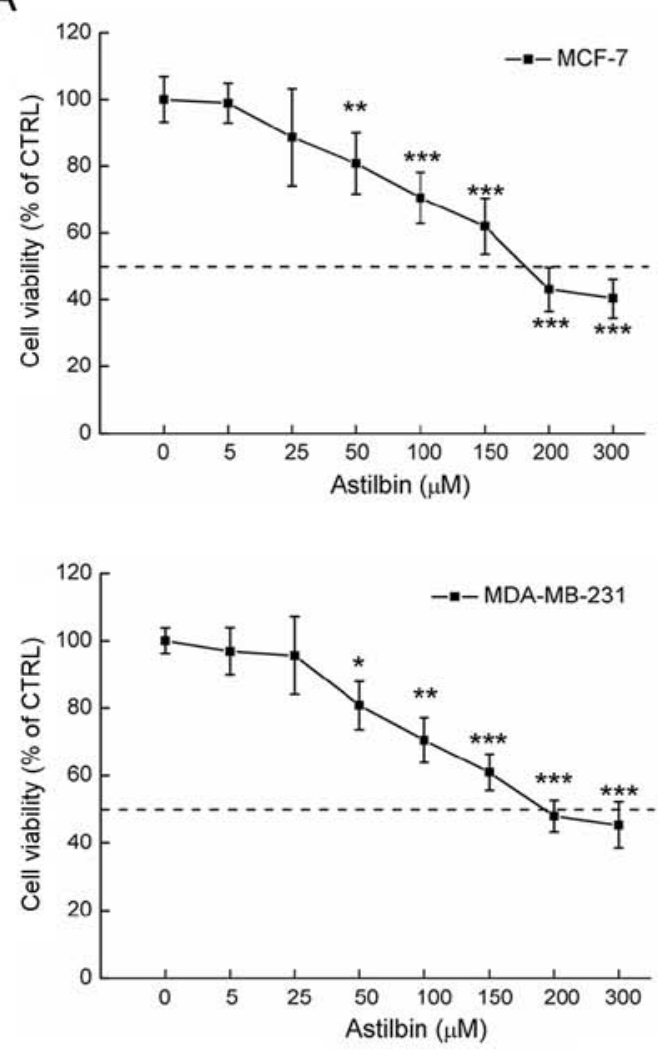

B

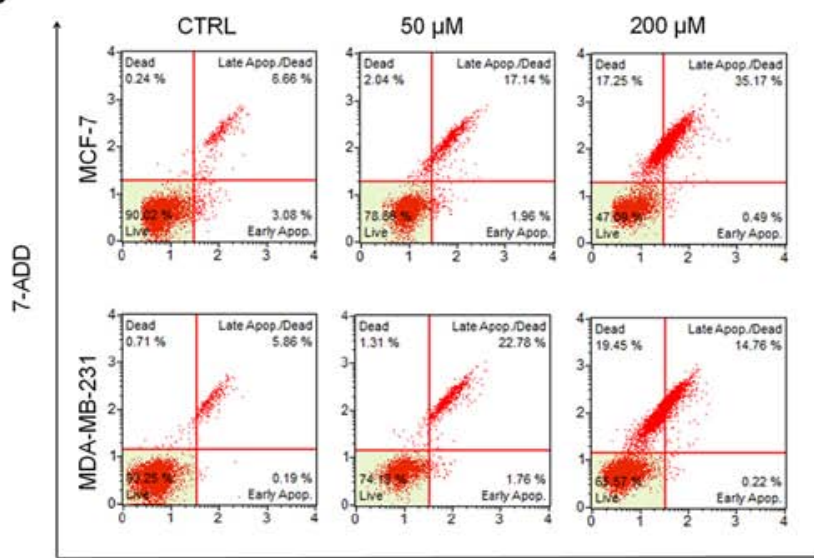

Annexin V-FITC

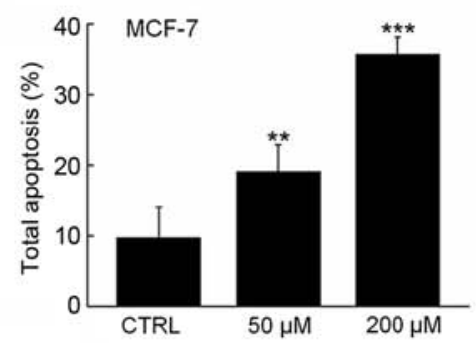

C
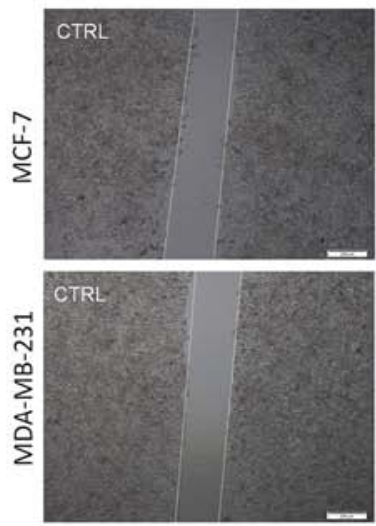
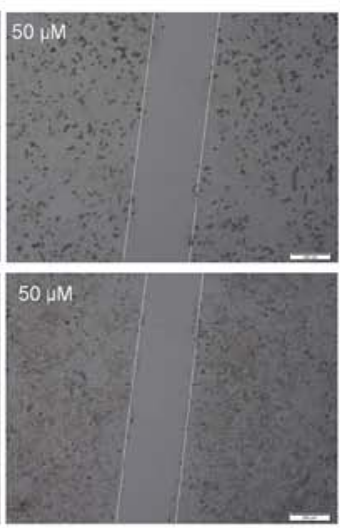
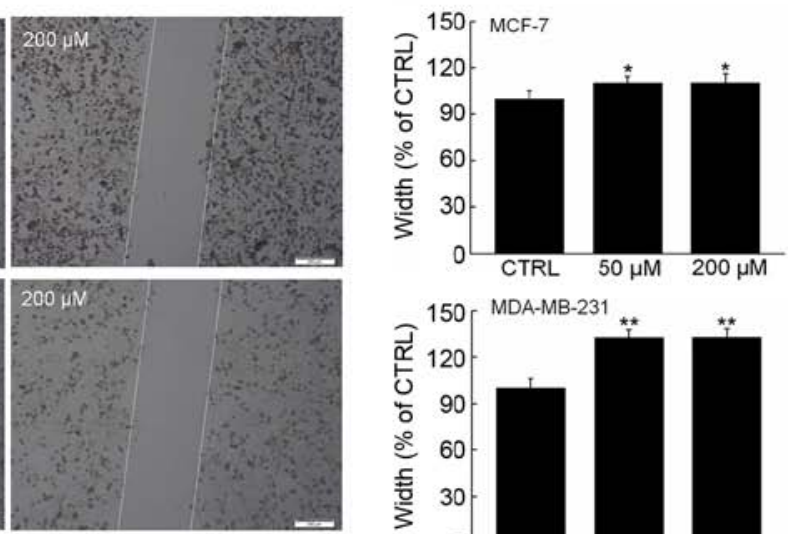

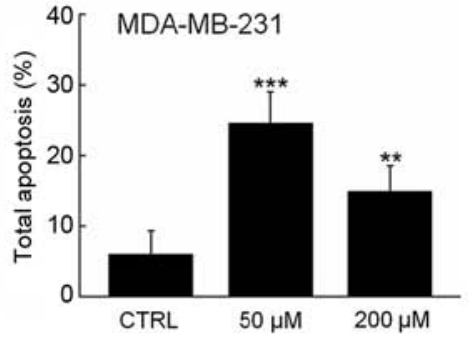

Figure 2. Intracellular toxicity of astilbin in MDA-MB-231 and MCF-7 cells. (A) MTT assay demonstrated that astilbin dose-dependently suppressed the cell viability after 24-h exposure (n=10). (B) Fow cytometry assay demonstrated that astilbin at doses of 50 and $200 \mu \mathrm{M}$ caused apoptosis in MDA-MB-231 and MCF-7 cells after 24-h exposure (n=6). (C) Astilbin inhibited the migration ability of MDA-MB-231 and MCF-7 cells after 24-h incubation, as observed via a wound healing assay (magnification, $\mathrm{x} 10$; scale bar, $200 \mu \mathrm{m} ; \mathrm{n}=6$ ). The distances of migrating cells were quantified using ImageJ software. Data are expressed as the mean \pm standard deviation. ${ }^{*} \mathrm{P}<0.05,{ }^{* *} \mathrm{P}<0.01$ and ${ }^{* * * *} \mathrm{P}<0.001$, vs. non-treated cells. CTRL, control.

Biochemical assays. Blood was collected from the caudal vein of each mouse prior to euthanasia. The levels of alanine aminotransferase (ALT) and aspartate aminotransferase (AST) in the serum were measured using commercial diagnostic kits (Nanjing Jiancheng Institute of Biotechnology Co., Ltd., Nanjing, China).

Histopathological examination. The liver, spleen and kidney tissues were fixed in $4 \%$ paraformaldehyde and subjected to histopathological examination using hematoxylin and eosin staining, as described in our previous study (15).
Western blot analysis. Samples of $4 \times 10^{5}$ cells per well were plated into 6-well plates, and treated with astilbin at the doses of 50 and $200 \mu \mathrm{M}$ for $24 \mathrm{~h}$. The treated cells and collected tumor tissues from the nude mice were lysed by radioimmunoprecipitation assay buffer containing $1 \%$ protease inhibitor cocktail (Sigma-Aldrich; Merck KGaA). Subsequent to protein concentration detection, $12 \%$ sodium dodecyl sulfate-polyacrylamide gel electrophoresis was used to separate the protein samples $(40 \mu \mathrm{g})$, which were further transferred electrophoretically onto $0.45-\mu \mathrm{m}$ nitrocellulose membranes (Bio Basic, Inc., Markham, ON, USA). 
A
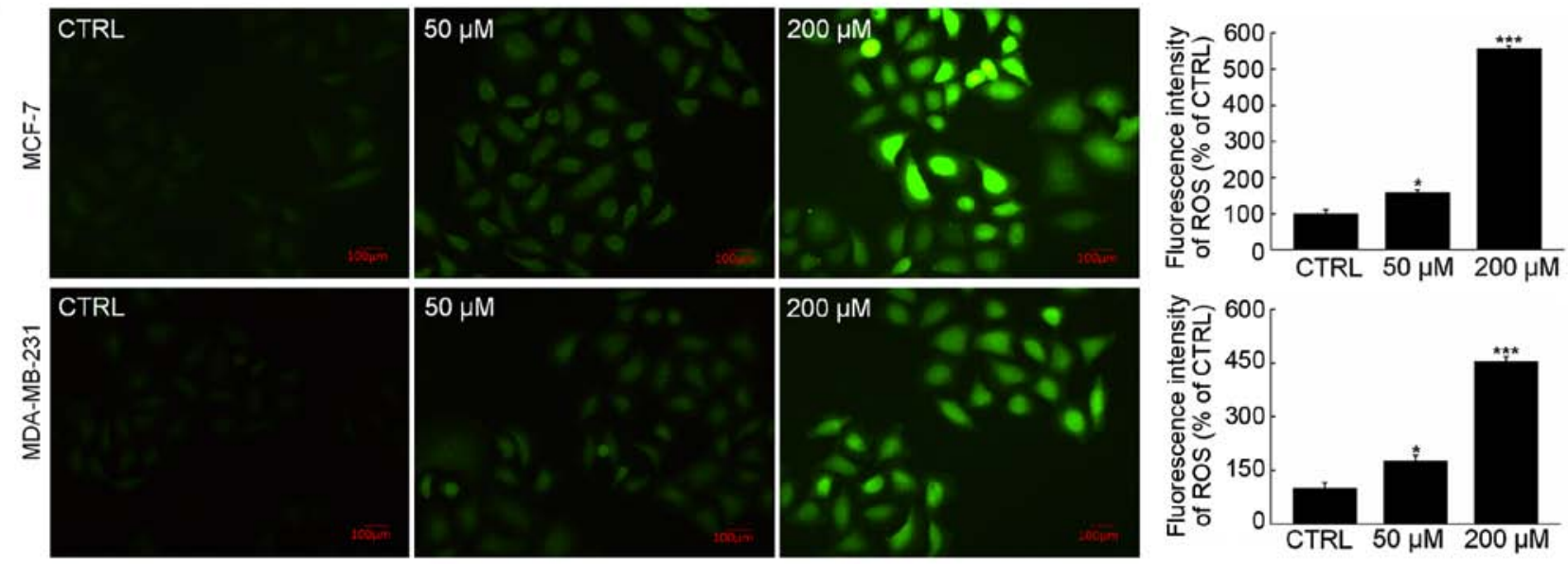

B
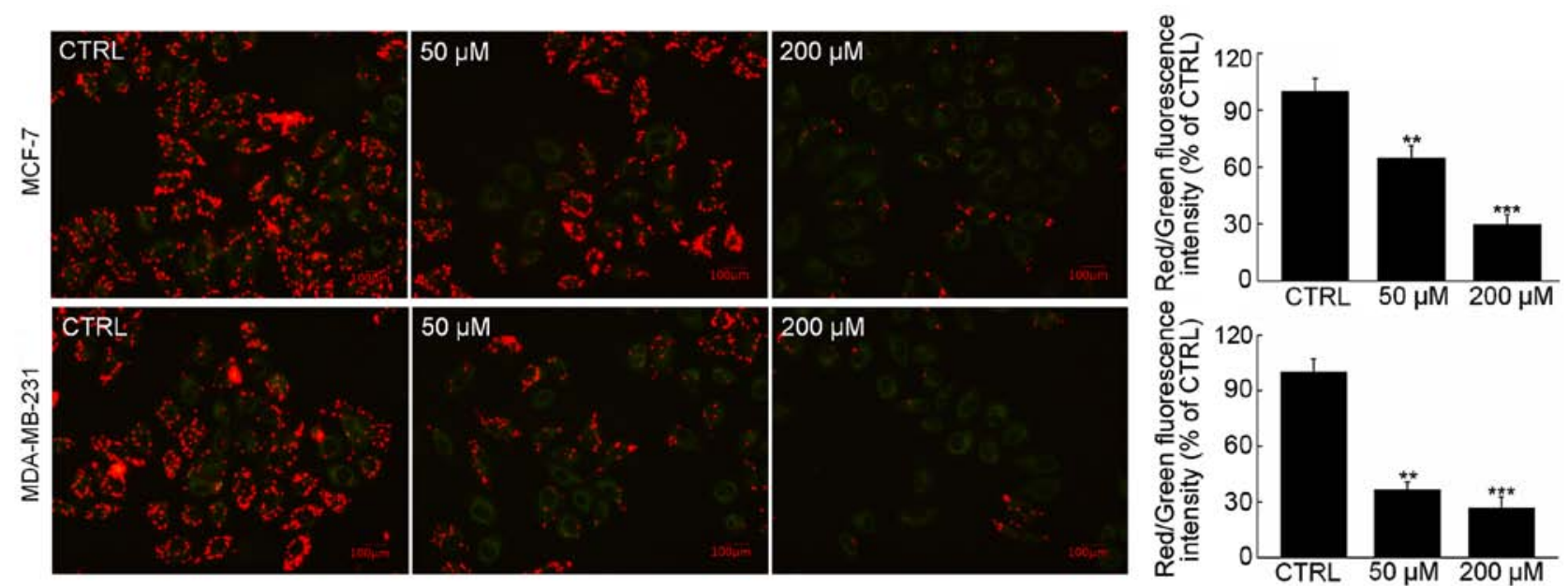

Figure 3. Astilbin caused the (A) accumulation of intracellular ROS levels and (B) dissipation of MMP in MCF-7 and MDA-MB-231 cells after 24-h exposure (magnification, x20; scale bar, $100 \mu \mathrm{m}$ ). The quantification data for ROS and MMP were expressed as the green fluorescence intensity or the ratio of red to green fluorescence intensity, respectively. Data are expressed as the mean \pm standard deviation $(n=6) .{ }^{*} \mathrm{P}<0.05,{ }^{* *} \mathrm{P}<0.01$ and ${ }^{* * * *} \mathrm{P}<0.001$, vs. non-treated cells. ROS, reactive oxygen species; MMP, mitochondrial membrane potential; CTRL, control.

The membranes were blotted with primary antibodies, including B-cell lymphoma 2 (Bcl-2; cat. no. 3498), Bcl-2associated X protein (Bax; cat. no. 14796), cleaved caspase-3 (cat. no. 9661), -8 (cat. no. 8592) and -9 (cat. no. 9509) and glyceraldehyde-3-phosphate dehydrogenase (GAPDH; cat. no. 5174) (all purchased from Santa Cruz Biotechnology, Inc., Dallas, TX, USA) at $4^{\circ} \mathrm{C}$ for $12 \mathrm{~h}$. The dilution of all primary antibodies was 1:2,000. After three washes with Tris-buffered saline/Tween-20 buffer, the membranes were then incubated with horseradish peroxidase-conjugated secondary antibodies (cat. nos. 7074 and 7076; Santa Cruz Biotechnology, Inc.) at a dilution of 1:3,000 for $4 \mathrm{~h}$ at room temperature. Chemiluminescence was performed using ECL detection kits (GE Healthcare Life Sciences, Little Chalfont, UK). ImageJ software was used to detect the intensity of the bands via scanning densitometry.

Statistical analysis. All experimental data in the present study are expressed as the mean \pm standard deviation. The statistical data were analyzed using one-way analysis of variance, followed by post hoc multiple comparisons (Duncan's multiple range test) using the SPSS version 16.0 software (SPSS, Inc.,
Chicago, IL, USA). A value of $\mathrm{P}<0.05$ was considered to denote a statistically significant difference.

\section{Results}

Astilbin suppresses the proliferation and migration, and enhances the apoptosis of breast carcinoma cells. Astilbin reduced the viability of both MDA-MB-231 and MCF-7 cells in a dose-dependent manner ( $\mathrm{P}<0.001$ at $300 \mu \mathrm{M} ; \mathrm{Fig}$. 2A). The half maximal inhibitory concentration $\left(\mathrm{IC}_{50}\right)$ values of astilbin for MDA-MB-231 and MCF-7 cells for a 24-h exposure were approximately 167.9 and $191.6 \mu \mathrm{M}$, respectively (Fig. 2A). The percentages of cell apoptosis after 24-h exposure to $50 \mu \mathrm{M}$ astilbin were $>19.1 \%(\mathrm{P}<0.01)$ and $>24.5 \%(\mathrm{P}<0.001)$ in MCF-7 cells and MDA-MB-231, respectively (Fig. 2B). Furthermore, the effects of astilbin on the migration ability of the breast carcinoma cells were investigated using a wound healing assay. In contrast to the non-treated cells, the cells treated with 24-h exposure to astilbin at the doses of 50 and $200 \mu \mathrm{M}$ exhibited significantly reduced migration into the wound area (Fig. 2C), indicating the suppressed migration abilities of the breast carcinoma cells. 
A

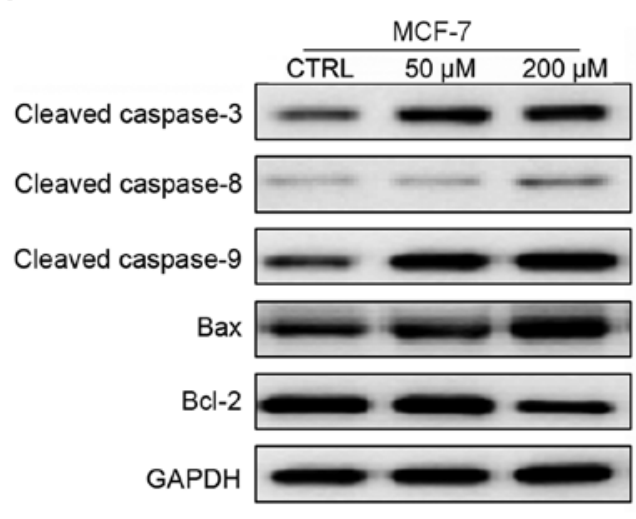

B

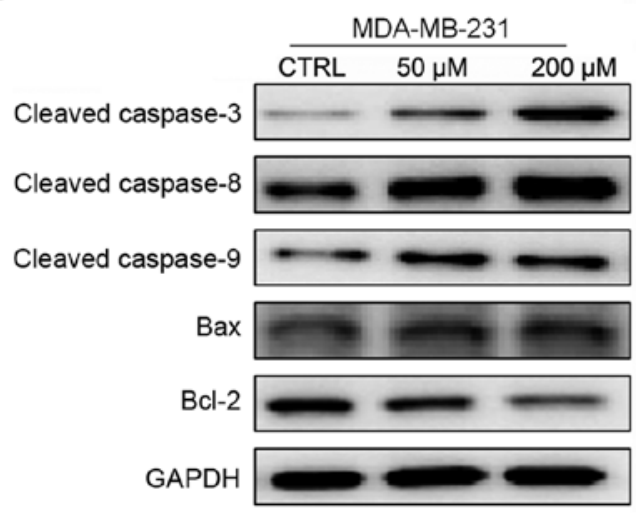

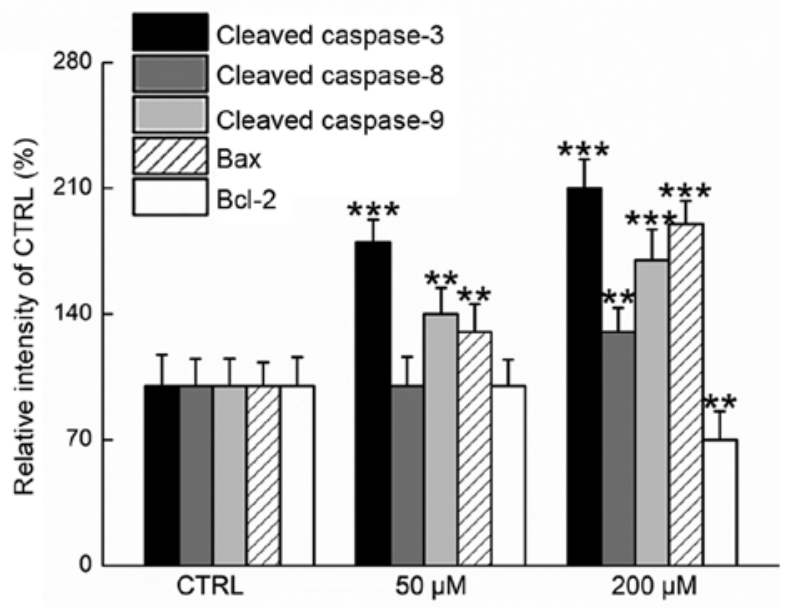

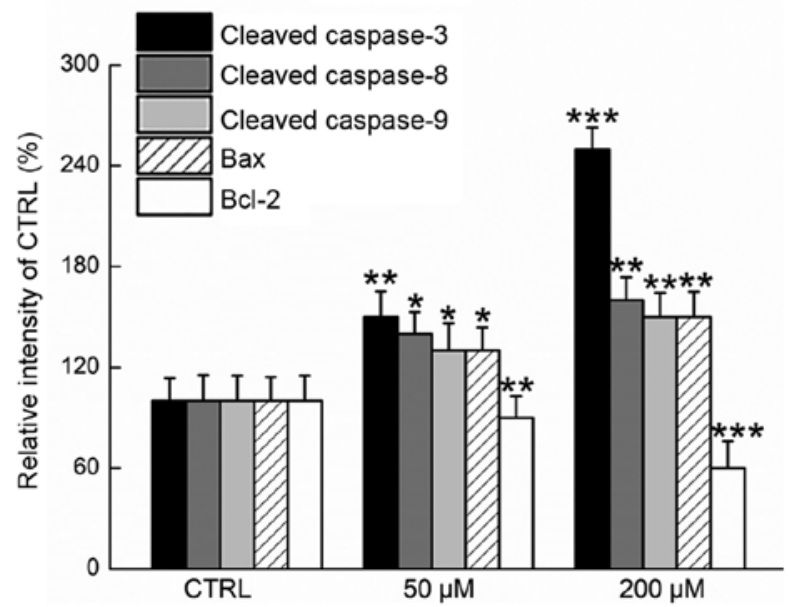

Figure 4. Astilbin enhanced the expression levels of Bax and cleaved caspase-3, -8 and -9 , and reduced the expression levels of Bcl-2 in (A) MCF-7 and (B) MDA-MB-231 cells after 24-h exposure, as determined via western blot analysis. The protein expression levels were quantified by densitometry analysis and normalized to the corresponding GAPDH levels. Data are expressed as the mean \pm standard deviation $(\mathrm{n}=6) .{ }^{*} \mathrm{P}<0.05,{ }^{* * *} \mathrm{P}<0.01$ and ${ }^{* * * *} \mathrm{P}<0.001$, vs. nontreated cells. Bcl-2, B-cell lymphoma 2; Bax, Bcl-2-associated X protein; CTRL, control.

Astilbin modulates mitochondrial function, and the expression levels of anti- and pro-apoptotic proteins. Intracellular levels of ROS not only influence mitochondrial function, but also serve an important role during cell apoptosis (14). Compared with the non-treated cells, the treated cells exhibited an enhanced green fluorescence intensity (Fig. 3A), suggesting the potential role of astilbin in promoting ROS production. In addition, treatment with astilbin at $200 \mu \mathrm{M}$ caused a statistically significant accumulation of ROS by $>5$-fold in MDA-MB-231 and MCF-7 cells $(\mathrm{P}<0.001$; Fig. 3A).

JC-1 staining was also performed to investigate the regulatory effect of astilbin on the MMP in the breast carcinoma cells, which is an indicator of mitochondrial function. Incubation with astilbin for $12 \mathrm{~h}$ strongly reduced the MMP in breast carcinoma cells, as indicated by the increased green fluorescence intensity and reduced red fluorescence intensity (Fig. 3B). Compared with the control cells, astilbin resulted in an approximately 50\% reduction in the ratio of red/green fluorescence intensity in the treated breast carcinoma cells ( $\mathrm{P}<0.001$; Fig. 3B).

Members of the caspase family, which are considered as critical participants in intrinsic and extrinsic mitochondrial signaling, were analyzed in the present study via western blot assay. It was observed that astilbin significantly increased the expression levels of cleaved caspases $-3,-8$ and -9 in MDA-MB-231 and MCF-7 cells after 24-h exposure ( $\mathrm{P}<0.05$; Fig. 4). Furthermore, Bax and Bcl-2 levels were examined, since the ratio of Bax and Bcl-2 serves as an important index for mitochondrial function (15). Compared with the control cells, 24-h exposure to astilbin resulted in a significant increase in Bax expression levels and marked suppression of Bcl-2 expression levels ( $\mathrm{P}<0.05$; Fig. 4). All these data conclusively confirmed that astilbin induced intracellular toxicity in breast carcinoma cells through the modulation of mitochondrial function.

Astilbin suppresses MCF-7 xenograft tumor growth in nude mice, and regulates the expression of anti-and pro-apoptotic proteins. In tumor xenograft male BALB/c nude mice, $20 \mathrm{mg} / \mathrm{kg}$ astilbin was intraperitoneally injected every other day for 14 days. The tumor growth was evidently suppressed by astilbin as compared with that observed in the control mice (Fig. 5A and B). The inhibitory activities of astilbin on tumor growth became apparent from day 7 , and caused $>6$-fold inhibition of the MCF-7 xenograft tumor growth by the day 15 
A

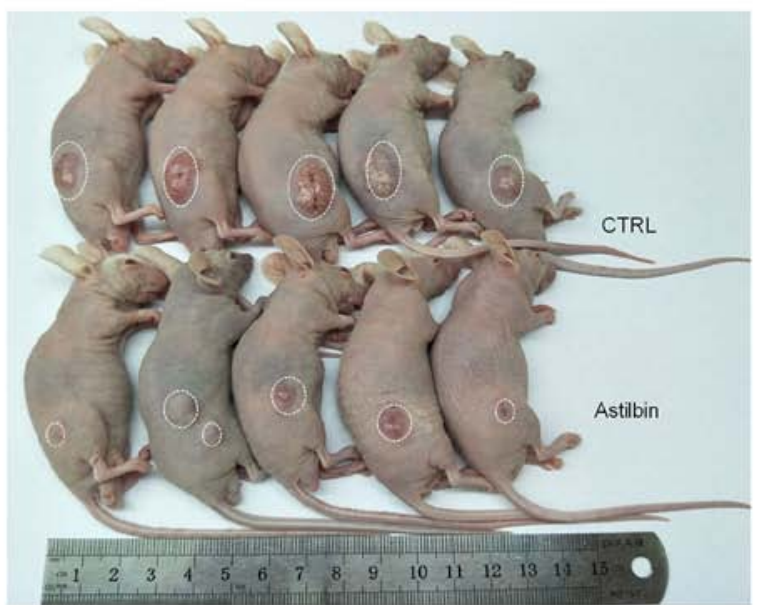

C

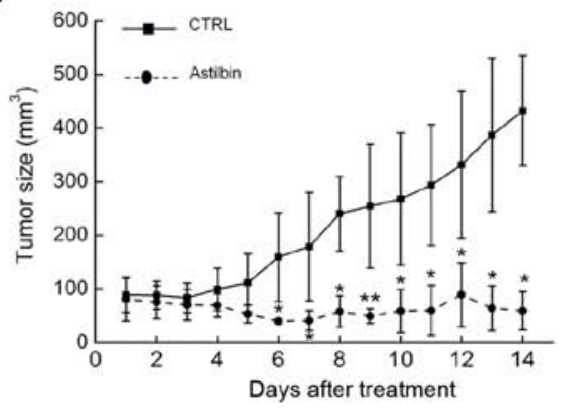

B

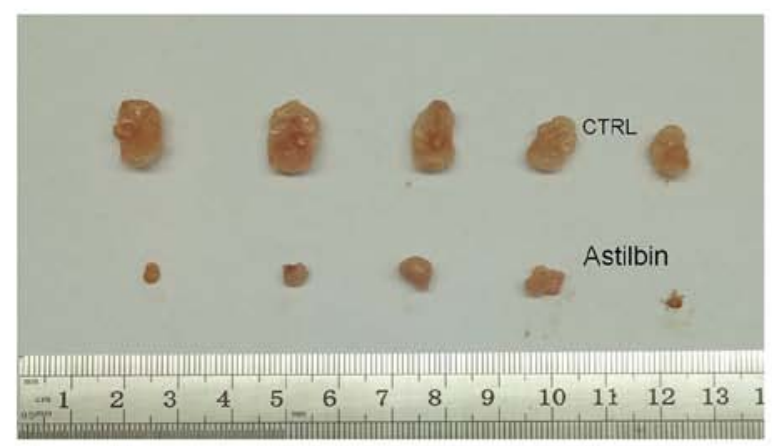

D

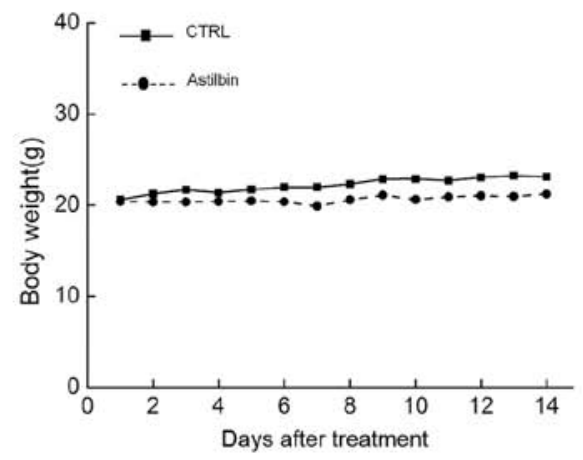

$E$
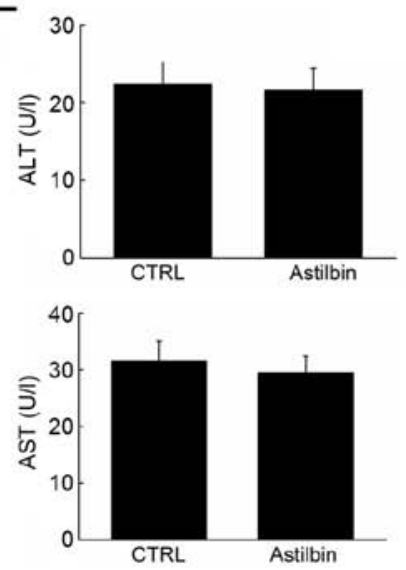

$\mathrm{F}$
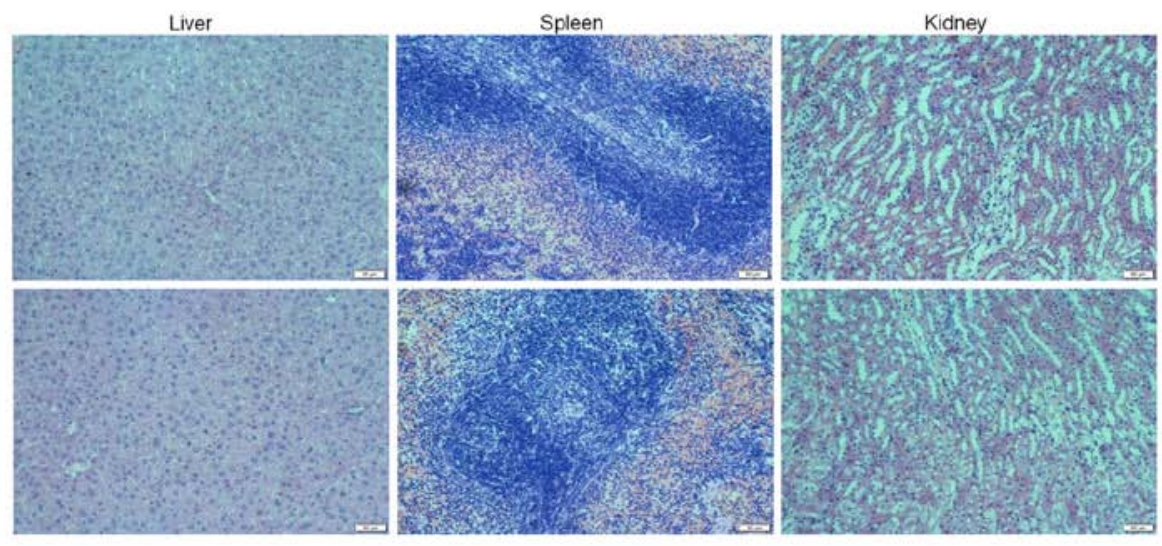

Figure 5. Astilbin suppressed the MCF-7-xenografted tumor growth in nude mice without influencing their body weight and organ function following injection at $20 \mathrm{mg} / \mathrm{kg}$ every other day for 14 days. (A) Tumor-possessing nude mice, (B) tumor tissue specimens, and (C) tumor growth curves of MCF-7-xenografted nude mice in the control and astilbin-treated groups. Tumor sizes were measured every two days. (D) Mean body weight of the control and astilbin-treated mice $(n=5)$. Data are expressed as the mean \pm standard deviation $(n=5)$. $P<0.05$ and ${ }^{* *} \mathrm{P}<0.01$, vs. vehicle group. (E) Astilbin failed to influence the serum levels of AST and ALT in nude mice compared with those in the control group. (F) Organ function, including liver, spleen and kidney function, of the control and astilbin-treated mice was analyzed by hematoxylin and eosin staining $(n=5)$. AST, aspartate aminotransferase; ALT, alanine aminotransferase; CTRL, control.

(P<0.05; Fig. 5C). However, astilbin had no significant influence on the body weight of mice (Fig. 5D), serum levels of AST and ALT (Fig. 5E), or organ function, including the liver, spleen and kidneys of the mice, (Fig. 5F), suggesting its safety for mouse treatment.

The expression levels of anti- and pro-apoptotic proteins in the tumor tissues were also measured. The results demonstrated that 14-day astilbin treatment significantly enhanced the expression levels of Bax and cleaved caspase-3, -8 and -9 , and reduced the expression levels of Bcl-2 in the tumor tissues of nude mice ( $\mathrm{P}<0.01$; Fig. 6$)$.

\section{Discussion}

The anti-inflammatory and anti-oxidant properties of astilbin have been widely studied $(16,18)$; however, its pro-apoptotic 

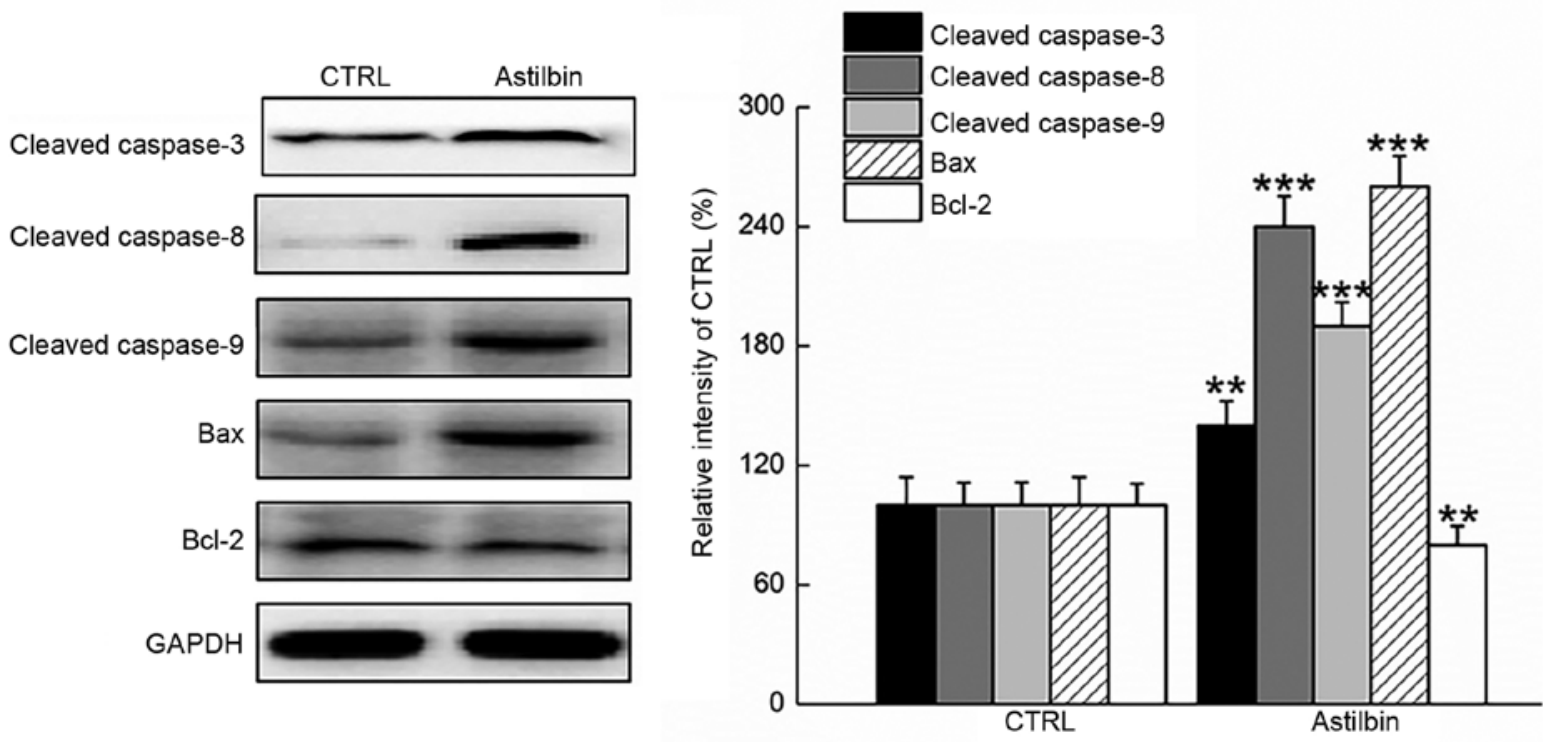

Figure 6. Astilbin enhanced the expression levels of Bax and cleaved caspase- $3,-8$ and -9 , and reduced the expression levels of Bcl-2 in the MCF-7-xenografted tumor of nude mice $(n=5)$. Protein expression levels were quantified by densitometry analysis and normalized to the corresponding GAPDH levels. Data are expressed as the mean \pm standard deviation $(\mathrm{n}=5)$. ${ }^{* * *} \mathrm{P}<0.01$ and ${ }^{* * * *} \mathrm{P}<0.001$, vs. control group. Bcl-2, B-cell lymphoma 2; Bax, Bcl-2-associated $\mathrm{X}$ protein; CTRL, control.

activities have not been fully elucidated. To the best of our knowledge, only one previous study examined the pro-apoptotic effect of Smilax glabra Roxb., which contains astilbin among its main chemical constituents, and reported that it induced apoptosis in hepatoma cell lines via modulation of the mitochondrial caspase-dependent apoptotic pathway (20). In the present study, the anti-breast cancer effects of astilbin were successfully confirmed in MDA-MB-231 and MCF-7 cells, and MCF-7-xenografted tumor nude mice. It was also verified that the caspase-dependent mitochondrial apoptotic pathway was involved in this process.

The safety of natural products has raised concerns among the medical community and the public (25). In the in vivo experiments conducted in the present study, astilbin did not influence the body weight of animals, serum levels of AST and ALT, or organ function (including the liver, spleen and kidney function), indicating its safety for use in animal experiments.

Astilbin also significantly reduced the viability, suppressed the migration ability, and increased the apoptosis rate of MDA-MB-231 and MCF-7 cells after 24-h exposure. Apoptosis, a physiological cell suicide mechanism, leads to cellular self-destruction characterized by chromatin condensation, distinct morphologic alterations and the formation of apoptotic bodies (26). The intrinsic apoptotic pathway is controlled by the mitochondria. The dissipation of MMP, which can serve as an index for mitochondrial apoptosis, is responsible for the loss of function in the mitochondria $(27,28)$. Following this process, mitochondrial permeability transition pores (MPTPs) are opened; consequently, pro-apoptotic molecules are released from the mitochondria, and the caspase family and other catabolic enzymes are activated (29). In the current study, astilbin incubation resulted in a marked reduction of MMP and overaccumulation of intracellular ROS in MDA-MB-231 and MCF-7 cells. It has previously been confirmed that the overaccumulation of ROS is linked to mitochondrial function, involving a short feedback loop (6). Increased levels of intracellular ROS facilitate the opening of MPTPs, which leads to further ROS release from the mitochondria into the cytoplasm (30). In the present study, it was observed that astilbin reduced the expression levels of $\mathrm{Bcl}-2$, and enhanced the expression levels of Bax, not only in human breast carcinoma cells, but also in MCF-7-xenografted tumor tissues. Bcl-2 and Bax are classic Bcl-2 family members, located in the outer mitochondrial membrane (31). The Bcl-2/ Bax heterodimer that is formed by interaction between the two proteins is involved in regulating the MMP (32). Previously, liquiritigenin was demonstrated to induce apoptosis in hepatoma carcinoma cells via MAPK-mediated mitochondrial apoptosis, partly via regulation of the expression levels of $\mathrm{Bcl}-2$ and Bax (33). This supports the findings of present study suggesting that astilbin exhibits anti-breast cancer properties via regulation of the mitochondrial or intrinsic apoptotic pathways.

Furthermore, astilbin was found to enhance the expression levels of cleaved caspase- $3,-8$ and -9 in MDA-MB-231 and MCF-7 cells, which is consistent with the earlier finding that mitochondrial function contributes to the activation of caspase (34). Caspase-8, mainly located in the mitochondria, can cleave itself to adopt the fully activated form, which results in the activation of Bid protein, leading to the dissipation of MMP (35-37). Consequently, cytochrome $c$, which is released from the mitochondria, helps to activate caspase-9 (38). Finally, caspase-3, the essential factor for the execution of the apoptotic program (39), is activated by cleaved caspase- 8 and -9 via proteolytic cleavage (40). In fact, this process is also involved in the feedback loop between ROS accumulation and mitochondrial function. Taken together, the activation of caspases (caspase-3, -8 and -9) contributes to the astilbin-mediated apoptosis of breast carcinoma cells. 
In the present study, male nude mice bearing MCF-7xenografted tumors were used to assess the pro-apoptotic activity of astilbin. However, previous research suggests that it is better to develop this model in female nude mice, which can provide sufficient levels of estrogen to help the tumor growth (41). The present study only aimed to investigate the pro-apoptotic activities of astilbin, and therefore avoided the influence of estrogen hormones during this process, since another separated experiment found that astilbin exhibited regulatory activities on estrogen hormones in healthy female Balb/c mice (unpublished data).

In conclusion, the present experimental study confirmed the anti-cancer effects of astilbin in breast carcinoma cells and nude mice bearing MCF-7-xenografted tumors. Astilbin enhanced the activation of caspase-3, -8 and -9 , and caused the overaccumulation of intracellular ROS and the dissipation of MMP, which led to apoptosis. The caspase-dependent mitochondrial pathway is, at least partially, involved in this process. These findings provide pharmacological support for astilbin as a candidate agent for breast cancer treatment.

\section{Acknowledgements}

Not applicable.

\section{Funding}

This study was supported by the Special Project of Industrial Technology Research and Development in Jilin Province (grant no. 2013-779).

\section{Availability of data and materials}

All data generated and analyzed during the present study are included in this published article.

\section{Authors' contributions}

SZ designed the experiments, wrote and revised the manuscript. $\mathrm{XS}$ drafted the manuscript and performed the experiments. $\mathrm{HZ}$ and $\mathrm{YZ}$ performed the experiments. QY analyzed the data. All authors read and approved the final manuscript and agreed to be accountable for all aspects of the work in ensuring that questions related to the accuracy or integrity of any part of the work are appropriately investigated and resolved.

\section{Ethics approval and consent to participate}

The experimental animal study was approved by the Changchun Medical College (approval no. CCMC2016-1201; Changchun, China) and the Second Hospital of Jilin University (Changchun, China).

\section{Patient consent for publication}

Not applicable.

\section{Competing interests}

The authors declare that they have no competing interests.

\section{References}

1. Hosseini BA, Pasdaran A, Kazemi T, Shanehbandi D, Karami H, Orangi $\mathrm{M}$ and Baradaran B: Dichloromethane fractions of Scrophularia oxysepala extract induce apoptosis in MCF-7 human breast cancer cells. Bosn J Basic Med Sci 15: 26-32, 2015.

2. Jemal A, Bray F, Center MM, Ferlay J, Ward E and Forman D: Global cancer statistics. CA Cancer J Clin 61: 69-90, 2011.

3. Nakagawa S, Shiraishi T, Kihara S and Tabuchi K: Detection of DNA strand breaks associated with apoptosis in human brain tumors. Virchows Arch 427: 175-179, 1995.

4. Joselin AP, Schulze-Osthoff K and Schwerk C: Loss of Acinus inhibits oligonucleosomal DNA fragmentation but not chromatin condensation during apoptosis. J Biol Chem 281: 12475-12484, 2006.

5. Shi H, Kwok RT, Liu J, Xing B, Tang BZ and Liu B: Real-time monitoring of cell apoptosis and drug screening using fluorescent light-up probe with aggregation-induced emission characteristics. J Am Chem Soc 134: 17972-17981, 2012.

6. Circu ML and Aw TY: Reactive oxygen species, cellular redox systems, and apoptosis. Free Radic Biol Med 48: 749-762, 2010.

7. Doley J, Singh LV, Kumar GR, Sahoo AP, Saxena L, Chaturvedi U, Saxena S, Kumar R, Singh PK, Rajmani RS, et al: Canine parvovirus type 2a (CPV-2a)-induced apoptosis in MDCK involves both extrinsic and intrinsic pathways. Appl Biochem Biotechnol 172: 497-508, 2014.

8. Tyagi N, Ovechkin AV, Lominadze D, Moshal KS and Tyagi SC: Mitochondrial mechanism of microvascular endothelial cells apoptosis in hyperhomocysteinemia. J Cell Biochem 98: 1150-1162, 2006

9. Liu X, Wang J, Lu C, Zhu C, Qian B, Li Z, Liu C, Shao J and Yan J: The role of lysosomes in BDE 47-mediated activation of mitochondrial apoptotic pathway in HepG2 cells. Chemosphere 124: 10-21, 2015.

10. Boatright KM, Renatus M, Scott FL, Sperandio S, Shin H, Pedersen IM, Ricci JE, Edris WA, Sutherlin DP, Green DR, et al: A unified model for apical caspase activation. Mol Cell 11: 529-541, 2003.

11. Kroemer G, Dallaporta B and Resche-Rigon M: The mitochondrial death/life regulator in apoptosis and necrosis. Annu Rev Physiol 60: 619-642, 1998.

12. Chen R, Liu S, Piao F, Wang Z, Qi Y, Li S, Zhang D and Shen J: 2,5-hexanedione induced apoptosis in mesenchymal stem cells from rat bone marrow via mitochondria-dependent caspase-3 pathway. Ind Health 53: 222-235, 2015.

13. Zhou Y, Guo Z, Meng Q, Lu J, Wang N, Liu H, Liang Q, Quan Y, Wang D and Xie J: Cordycepin affects multiple apoptotic pathways to mediate hepatocellular carcinoma cell death. Anticancer Agents Med Chem 17: 143-149, 2017.

14. Wang D, Wong HK, Feng YB and Zhang ZJ: 18beta-glycyrrhetinic acid induces apoptosis in pituitary adenoma cells via ROS/MAPKs-mediated pathway. J Neurooncol 116: 221-230, 2014.

15. Zhang X, Chen Y, Cai G, Li X and Wang D: Carnosic acid induces apoptosis of hepatocellular carcinoma cells via ROS-mediated mitochondrial pathway. Chem Biol Interact 277: 91-100, 2017.

16. Wang SW, Xu Y, Weng YY, Fan XY, Bai YF, Zheng XY, Lou LJ and Zhang F: Astilbin ameliorates cisplatin-induced nephrotoxicity through reducing oxidative stress and inflammation. Food Chem Toxicol 114: 227-236, 2018.

17. Wang J, Zhao $\mathrm{Y}$ and $\mathrm{Xu} \mathrm{Q}$ : Astilbin prevents concanavalin A-induced liver injury by reducing TNF-alpha production and T lymphocytes adhesion. J Pharm Pharmacol 56: 495-502, 2004.

18. Wang M, Zhao J, Zhang $\mathrm{N}$ and Chen J: Astilbin improves potassium oxonate-induced hyperuricemia and kidney injury through regulating oxidative stress and inflammation response in mice. Biomed Pharmacother 83: 975-988, 2016.

19. Chen L, Lan Z, Zhou Y, Li F, Zhang X, Zhang C, Yang Z and Li P: Astilbin attenuates hyperuricemia and ameliorates nephropathy in fructose-induced hyperuricemic rats. Planta Med 77: 1769-1773, 2011.

20. Sa F, Gao JL, Fung KP, Zheng Y, Lee SM and Wang YT: Antiproliferative and pro-apoptotic effect of Smilax glabra Roxb. extract on hepatoma cell lines. Chem Biol Interact 171: 1-14, 2008.

21. Mosmann T: Rapid colorimetric assay for cellular growth and survival: Application to proliferation and cytotoxicity assays. J Immunol Methods 65: 55-63, 1983. 
22. Song J, Wang Y, Teng M, Zhang S, Yin M, Lu J, Liu Y, Lee RJ Wang D and Teng L: Cordyceps militaris induces tumor cell death via the caspase-dependent mitochondrial pathway in HepG2 and MCF-7 cells. Mol Med Rep 13: 5132-5140, 2016.

23. Lai H, Fu X, Sang C, Hou L, Feng P, Li X and Chen T: Selenadiazole derivatives inhibit angiogenesis-mediated human breast tumor growth by suppressing the VEGFR2-mediated ERK and AKT signaling pathways. Chem Asian J 13: 1447-1457, 2018.

24. Yang X, Ding Y, Xiao M, Liu X, Ruan J and Xue P: Anti-tumor compound RY10-4 suppresses multidrug resistance in MCF-7/ ADR cells by inhibiting PI3K/Akt/NF- $\mathrm{KB}$ signaling. Chem Biol Interact 278: 22-31, 2017

25. Gao L, Liu G, Kang J, Niu M, Wang Z, Wang H, Ma J and Wang X: Paclitaxel nanosuspensions coated with P-gp inhibitory surfactants: I. Acute toxicity and pharmacokinetics studies. Colloids Surf B Biointerfaces 111: 277-281, 2013.

26. Blanco GA, Bustamante J, Garcia M and Hajos SE: Hydrogen peroxide induces apoptotic-like cell death in coelomocytes of Themiste petricola (Sipuncula). Biol Bull 209: 168-183, 2005.

27. Hengartner MO: The biochemistry of apoptosis. Nature 407: 770-776, 2000

28. Galluzzi L, Vitale I, Kepp O, Séror C, Hangen E, Perfettini JL, Modjtahedi N and Kroemer G: Methods to dissect mitochondrial membrane permeabilization in the course of apoptosis. Methods Enzymol 442: 355-374, 2008

29. Bensassi F, Gallerne C, el Dein OS, Hajlaoui MR, Bacha H and Lemaire C: Mechanism of Alternariol monomethyl etherinduced mitochondrial apoptosis in human colon carcinoma cells. Toxicology 290: 230-240, 2011.

30. Wang $\mathrm{H}$ and $\mathrm{Xu} \mathrm{W}$ : Mito-methyl coumarin, a novel mitochondriatargeted drug with great antitumor potential was synthesized. Biochem Biophys Res Commun 489: 1-7, 2017.

31. Chan SL and Yu VC: Proteins of the bcl-2 family in apoptosis signalling: From mechanistic insights to therapeutic opportunities. Clin Exp Pharmacol Physiol 31: 119-128, 2004

32. Ding J, Mooers BH, Zhang Z, Kale J, Falcone D, McNichol J, Huang B, Zhang XC, Xing C, Andrews DW, et al: After embedding in membranes antiapoptotic Bcl-XL protein binds both Bcl-2 homology region 3 and helix 1 of proapoptotic Bax protein to inhibit apoptotic mitochondrial permeabilization. J Biol Chem 289: 11873-11896, 2014.
33. Wang D, Lu J, Liu Y, Meng Q, Xie J, Wang Z and Teng L: Liquiritigenin induces tumor cell death through mitogenactivated protein kinase- (MPAKs-) mediated pathway in hepatocellular carcinoma cells. Biomed Res Int 2014: 965316 2014.

34. Xin Y, Huang Q, Zhang P, Guo WW, Zhang LZ and Jiang G: Demethoxycurcumin in combination with ultraviolet radiation $B$ induces apoptosis through the mitochondrial pathway and caspase activation in A431 and HaCaT cells. Tumour Biol 39 $1010428317706216,2017$.

35. Schug ZT, Gonzalvez F, Houtkooper RH, Vaz FM and Gottlieb E: BID is cleaved by caspase- 8 within a native complex on the mitochondrial membrane. Cell Death Differ 18: 538-548, 2011

36. Hyun HB, Lee WS, Go SI, Nagappan A, Park C, Han MH, Hong SH, Kim G, Kim GY, Cheong J, et al: The flavonoid morin from Moraceae induces apoptosis by modulation of $\mathrm{Bcl}-2$ family members and Fas receptor in HCT 116 cells. Int J Oncol 46: 2670-2678, 2015

37. Lee JW, Park C, Han MH, Hong SH, Lee TK, Lee SH, Kim GY and Choi YH: Induction of human leukemia U937 cell apoptosis by an ethanol extract of Dendropanax morbifera Lev. through the caspase-dependent pathway. Oncol Rep 30: 1231-1238, 2013.

38. Wu Y, Zhang P, Yang H, Ge Y and Xin Y: Effects of demethoxycurcumin on the viability and apoptosis of skin cancer cells. Mol Med Rep 16: 539-546, 2017.

39. Porter AG and Jänicke RU: Emerging roles of caspase-3 in apoptosis. Cell Death Differ 6: 99-104, 1999.

40. Zhang WG, Liu XF, Meng KW and Hu SY: Puerarin inhibits growth and induces apoptosis in SMMC-7721 hepatocellular carcinoma cells. Mol Med Rep 10: 2752-2758, 2014.

41. Indran IR, Zhang SJ, Zhang ZW, Sun F, Gong Y, Wang X, Li J, Erdelmeier CA, Koch E and Yong EL: Selective estrogen receptor modulator effects of epimedium extracts on breast cancer and uterine growth in nude mice. Planta Med 80: 22-28, 2014. 